\title{
Lateral Nonuniformity Effects of Border Traps on the Characteristics of Metal-Oxide-Semiconductor Field-Effect Transistors Subjected to High-Field Stresses
}

\author{
Jen-Chou Tseng and Jenn-Gwo Hwu, Senior Member, IEEE
}

\begin{abstract}
The lateral nonuniformity (LNU) effects of border traps are studied by exploring both the high- and low-frequency characteristics in $\mathrm{N}$-type channel metal-oxide-semiconductor field-effect transistors. According to experimental data, the deterioration of nonuniformity is significantly enhanced at low frequencies. The cause may be due to the additional trapped charges of border traps (near-interface oxide traps) under the low-frequency measurement. This model is successfully simulated by the combination of low-frequency $C-V$ curves with the heavily and lightly damaged regions. Additionally, the double-peak charge-pumping current is observed in low-frequency measurements, which can further support our hypothesis that border-trap-enhanced LNU exists. Finally, the geometric effect of the polygate and the thickness effect of the gate oxide are also investigated for the nonuniformity issue.
\end{abstract}

Index Terms-Gate dielectrics, metal-oxide-semiconductor (MOS) devices, oxide traps, semiconductor device reliability.

\section{INTRODUCTION}

A NALOG circuits are very sensitive to stress-induced latent defects [1], [2]. If an environmental high-field stress is applied to an analog circuit device, the related parameter drift will impact the device performance and operation. The functionality of sensitive analog circuits (such as analog-to-digital converters, digital-to-analog converters, operational amplifiers, and analog computation circuits) depends on the accuracy of the device parameters. For instance, leakage currents from gates or channels may greatly increase the power consumption. An asymmetric stress on the input pair of a differential amplifier can cause input offset voltages [2]. In addition, a metal-oxidesemiconductor (MOS) capacitor such as a voltage-control varactor in the voltage-control oscillator can lose efficacy due to the capacitance-voltage $(C-V)$ distortion after stresses. Hence, the stress-induced leakage current, threshold voltage $\left(V_{T}\right)$ shift, mobility degradation, and $C-V$ distortion are important relia-

Manuscript received July 10, 2007; revised March 11, 2008. This work was supported by the National Science Council of Taiwan, R.O.C., under Contract NSC 95-2221-E-002-375. The review of this paper was arranged by Editor J. Suehle.

J.-C. Tseng was with the Department of Electrical Engineering and the Graduate Institute of Electronics Engineering, National Taiwan University, Taipei 106, Taiwan, R.O.C. He is now with Taiwan Semiconductor Manufacturing Company, Hsinchu 300, Taiwan, R.O.C.

J.-G. Hwu is with the Department of Electrical Engineering and the Graduate Institute of Electronics Engineering, National Taiwan University, Taipei 106, Taiwan, R.O.C. (e-mail: hwu@cc.ee.ntu.edu.tw).

Digital Object Identifier 10.1109/TED.2008.922489 bility concerns [3], [4]. Moreover, some localized damages can further introduce nonuniformity issues and impose a linearity problem on analog circuits.

Generally, oxide stress leads to a homogeneously distributed damage, which consists of stress-induced interface traps and positive trapped charges in the oxide. The correlation between interface state density and $V_{T}$ shift is easily obtained by using the charge-pumping technique. However, studies of the localized damage due to a high-field stress on the oxide are seldom discussed via low-frequency characterization. According to existing reports [5]-[8], lateral nonuniformity (LNU) studies are generally focused on the oxide-trapped charges using high-frequency measurements. Chim and Lim [7] studied the formation of LNU charges in MOS capacitors subjected to electrostatic discharge (ESD) impulse stresses. Huang and Hwu [6] investigated the LNU of effective oxide charges in MOS capacitors with aluminum oxide $\left(\mathrm{Al}_{2} \mathrm{O}_{3}\right)$ gate dielectrics using the high-frequency Terman's method, and a parallel capacitor model was constructed to simulate the LNU phenomenon. Nevertheless, these simulations may neglect the detailed effect and mechanism of traps under an inversion regime at low-frequency operations. As reported, the bulk oxide traps and near-interface oxide traps (border traps), as well as the interface traps, are generated during a high-field stress on gate oxides [9]-[14]. The generation of border traps can lead to an anomalous "hump" distortion in the inversion region of the quasi-static $C-V$ curve [12] due to the communication with the $\mathrm{Si}-\mathrm{SiO}_{2}$ interface traps. As the bias voltage changes under the low-frequency condition, these border traps can trap or detrap charges via a trap-to-trap tunneling mechanism to alter the electric field at the interface and impact the accuracy of device operation. In this paper, a set of experiments was performed to study the characteristic drift of MOS devices subjected to high-field oxide stresses, including high- and low-frequency $C-V$ and chargepumping measurements. An anomalous charge-pumping signal with double peaks was observed under a low-frequency measurement in the high-field-stressed devices, which we attributed the enhancement of LNU to the border-trapped charges. Furthermore, this enhancement of LNU effect is successfully simulated using the low-frequency $C-V$ curves with different shifts of flatband voltages based on the parallel-capacitor model. Finally, an explanation is proposed for the possible mechanism regarding the origin of border-trap-enhanced LNU effects. 


\section{EXPERIMENTS}

Experiments were performed on n-channel MOS fieldeffect transistors (NMOSFETs) with 14-nm-thick and 3.2-nmthin gate oxides, fabricated by a standard $0.18-\mu \mathrm{m}$ dual-gate complementary MOS technology. The NMOSFETs are finger shaped with p-type substrates. For the 14-nm samples, there are long- and short-channel MOSFETs that are being tested. The polygate's width and length for the long-channel MOSFET are 300 and $10 \mu \mathrm{m}$, respectively. On the other hand, the gate's width and length for the short-channel MOSFET are 200 and $0.6 \mu \mathrm{m}$, respectively. For the $3.2-\mathrm{nm}$ samples, the polygate's width and length are 150 and $0.16 \mu \mathrm{m}$, respectively. The gate oxide stresses were performed in the accumulation mode with one contact to the gate and another contact to the substrate. Note that the source and drain were kept floating during stressing. We used an HP-4156C semiconductor parameter analyzer to perform the dc high-field stresses and quasi-static $C-V$ characteristics. In addition, an HP-4284 precision $L C R$ meter was adopted to measure the high-frequency $(1 \mathrm{MHz})$ and lowfrequency $(1 \mathrm{kHz}$ or $100 \mathrm{~Hz}) C-V$ characteristics. The setup of charge-pumping measurements is similar to those in the previous studies [15]-[17], a pulse train of fixed amplitude $V_{A}$ from an HP-8110A pulse generator is applied to the gate, and the source and drain are connected to the ground, while the substrate current (charge-pumping current $I_{\mathrm{cp}}$ ) is measured. When the base-level voltage $V_{\text {base }}$ of the pulse is lower than the flatband voltage $V_{\mathrm{FB}}$ and the top-level voltage $V_{\mathrm{top}}$ of the pulse is higher than the threshold voltage $V_{t}$, the conventional chargepumping effect occurs. The applied frequencies were $1 \mathrm{MHz}$ and $100 \mathrm{~Hz}$ with a rise/fall time of $0.2 \mu \mathrm{s}$. Duty cycles were all $50 \%$. The $V_{A}$ values were 2 and $4 \mathrm{~V}$ for the 3.2- and 14-nm devices, respectively. Our experimental procedures are described as follows. First, the NMOSFETs were subjected to a dc high-field stress. After each 0.2-s dc stress, the stressing was interrupted. The $C-V$ and charge-pumping measurements were performed. This procedure was repeated until the device failed due to the oxide breakdown.

\section{RESUlTS AND DISCUSSION}

\section{A. High-Field Stress Induced Distortion Under Low-Frequency $C-V$ Measurements}

Since most interface traps cannot follow a high-frequency signal at $1 \mathrm{MHz}$, the $C-V$ curves measured at $1 \mathrm{MHz}$ can reveal the characteristics of bulk oxide charges. Additionally, as the frequency is less than $1000 \mathrm{~Hz}$, the border traps can lead to a rapid increase in charges recombined per cycle and trap charges near the $\mathrm{Si}-\mathrm{SiO}_{2}$ interface by trap-to-trap tunneling processes [11], [12]. The border traps dominate over the interface traps under the low-frequency condition, because the number of charges provided from interface traps is far less than those from border traps at low frequencies, as per previous publications' results [12]. Therefore, the low-frequency $(<1000 \mathrm{~Hz})$ and quasistatic $C-V$ curves can indicate the characteristics of border traps. Fig. 1(a)-(c) shows the $C-V$ characteristics of a largewidth long-channel NMOSFET subjected to a series of highfield dc stresses for the high-frequency, low-frequency, and

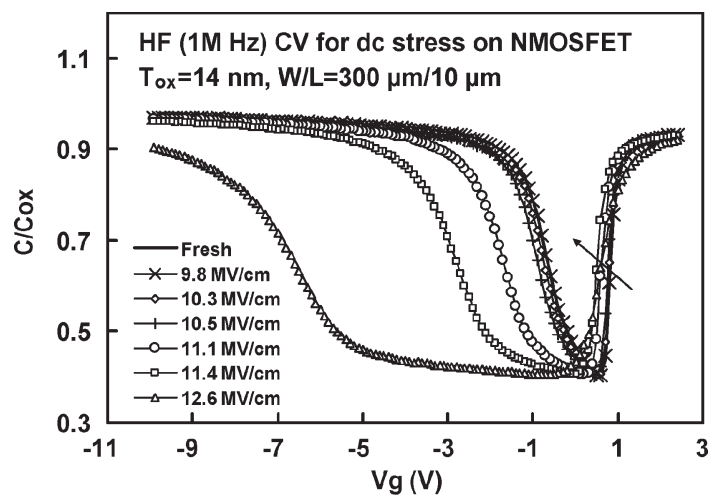

(a)

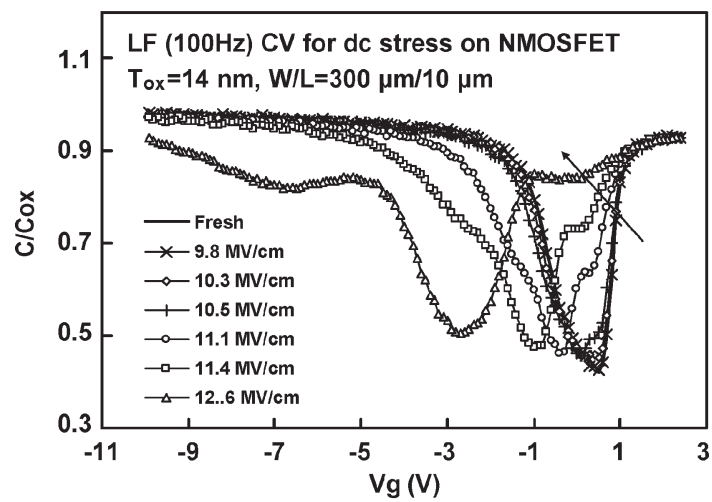

(b)

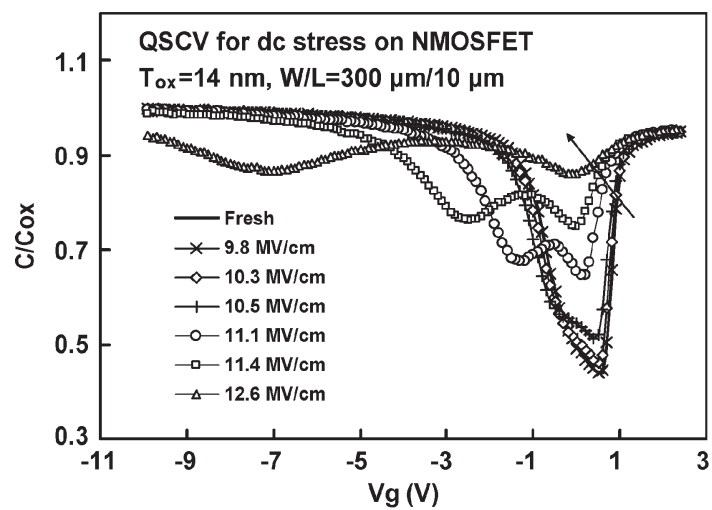

(c)

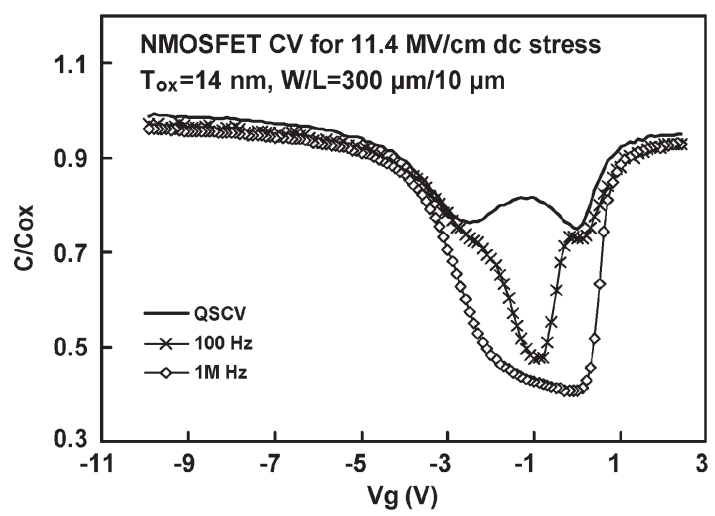

(d)

Fig. 1. (a) High-frequency (1 MHz), (b) low-frequency $(100 \mathrm{~Hz})$, and (c) quasi-static $C-V$ characteristics of a long-channel $(10 \mu \mathrm{m})$ NMOSFET subjected to a series of dc high-field stresses. (d) Comparison of $C-V$ curves over the high, low, and quasi-static frequencies. 
quasi-static frequency measurements, respectively. Fig. 1(d) shows the comparison of $C-V$ curves over the high, low, and quasi-static frequencies. As the figures reveal, the flatband voltage $V_{\mathrm{FB}}$ shift and $C-V$ curve distortion were caused by stresses and became more pronounced for a heavier stress. For the high-frequency $C-V$ curves, the oxide-trapped charges $Q_{\text {ot }}$ were positive in nature, as can be seen from the curve shift toward the negative gate voltage direction. On the other hand, for the $100 \mathrm{~Hz}$ and quasi-static $C-V$ curves, the slow interface and border traps were observed to trap charges and lead to serious distortions of curves. According to the previous studies [5]-[8], this kind of distortions in high-frequency $C-V$ curves may be due to the nonuniform spatial distribution of trapped charges in the gate oxide, and the NMOS capacitor can be modeled by two connected capacitors in parallel with identical oxide thicknesses and different flatband voltages. It is assumed that these two capacitors are independent and do not react with each other. Their flatband voltages are $V_{\mathrm{FB} 1}$ and $V_{\mathrm{FB} 2}$, and their area ratios are $A_{1}$ and $A_{2}\left(A_{1}+A_{2}=1\right)$. The synthesized capacitance is obtained by the following equation [7]:

$$
C=A_{1} \times C_{1}+A_{2} \times C_{2} .
$$

Additionally, the flatband voltage can be written as

$$
V_{\mathrm{FB}}=\phi_{\mathrm{ms}}-\frac{Q_{\mathrm{eff}}}{C_{\mathrm{ox}}}
$$

where $\phi_{\mathrm{ms}}$ is the work function difference between the gate metal and $\mathrm{Si}, C_{\text {ox }}$ is the oxide capacitance, $Q_{\text {eff }}$ is the summation of effective oxide-trapped charges $Q_{\mathrm{ot}}$, border-trapped charges $Q_{\mathrm{bt}}$, and interface-trapped charges $Q_{\text {it }}$. Hence, the different $V_{\mathrm{FB}}$ values indicate different amounts of $Q_{\text {eff }}$. It is noted that no contribution of interface-trap-induced capacitance $C_{\text {it }}$ is considered in these simulations, and that the $C-V$ curve's distortion is purely caused by the LNU of $Q_{\text {eff }}$.

The low-frequency $(100 \mathrm{~Hz}) C-V$ curves after stresses are extracted from Fig. 1(b) and simulated by the above model in Fig. 2. The simulation was performed to study the border trap's effect by the simplified model, assuming two connected capacitors in parallel for the heavily stressed MOS device. One is heavily damaged, and the other is lightly damaged. The ratio between them is assumed to be $1: 1$. To present the nonuniform damaged regions of the MOSFET, different $V_{\mathrm{FB}}$ shift values were added to the fresh $C-V$ curves. Then, these two curves were weighted and summed to match the LNU $C-V$ curve of the whole MOSFET subjected to stresses. The inserts in Fig. 2 show the extra contribution of trapped charges by the border traps. When the MOS capacitor is biased under the accumulation mode, the donor-like border traps contribute positive trapped charges and lead to a negative shift of $V_{\mathrm{FB}}$. On the other hand, when the MOS capacitor is biased under the inversion mode, the acceptor-like border traps contribute negative trapped charges and lead to a positive shift of $V_{\mathrm{FB}}$. As the figure shows, the combination of curves with $\Delta V_{\mathrm{FB}}=-1.4$ and $-0.2 \mathrm{~V}$ matches the measured $C-V$ curve well when the gate biased voltage is lower than $-0.7 \mathrm{~V}$. Furthermore, when the gate biased voltage ranges from -0.7 to $0.3 \mathrm{~V}$, the combination

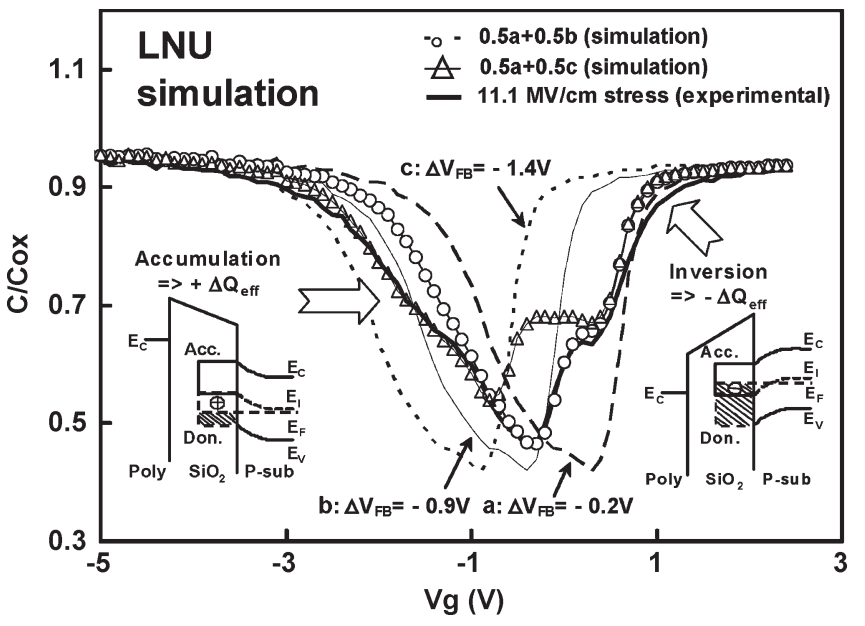

Fig. 2. Low-frequency $C-V$ curves of devices after stresses are extracted from (b) and simulated by the proposed model with enhanced LNU phenomenon. The inserts show the extra contribution of trapped charges by the slow border traps when the MOS capacitor is biased under accumulation and inversion modes.

of curves with $\Delta V_{\mathrm{FB}}=-0.9$ and $-0.2 \mathrm{~V}$ can entirely match the measured $C-V$ curve. Hence, a larger shift of $V_{\mathrm{FB}}$ caused by the donor-like $Q_{\mathrm{bt}}$ helps in matching the larger distortion curve in an accumulation regime. On the other hand, when the gate-biased voltage is larger than $0.3 \mathrm{~V}$, there is about a $0.1-\mathrm{V}$ shift of $V_{\mathrm{FB}}$ between the combination $\left(\Delta V_{\mathrm{FB}}=-0.9\right.$ and $-0.2 \mathrm{~V}$ ) and measured $C-V$ curves, which is caused by the additional acceptor-like $Q_{\mathrm{bt}}$ in an inversion regime. Therefore, good agreement is achieved by selecting appropriate combinations with different amounts of $Q_{\text {eff }}$, which cause different $V_{\mathrm{FB}}$ shifts under the different gate-biased voltages. By assuming that the $Q_{\text {eff }}$ generation by border traps is proportional to the damage caused by the corresponding stress current, it is revealed that these nonuniform trapped charges are caused by the nonuniform distribution of stress currents. Therefore, we can attribute the LNU effect to border traps in the simulation at low frequencies $(<1000 \mathrm{~Hz})$.

\section{B. Geometric Effect of High Field Stress}

In previous publications regarding high-field ESD stresses on gate oxides, Huang and Hwu [6] attributed the LNU effect to the localized injection of avalanche hot carriers with the accompanying impact ionization within the oxide. Leroux et al. [18] proposed that the gate oxide tunneling current is homogeneous for a low-level ESD but is crowding during a high-level ESD. Additionally, the current crowding is due to the high resistance of a narrow polysilicon gate structure. These assumptions may also be true under the dc high-field stress on oxide. Fig. 3(a)-(c) shows the $C-V$ characteristics of short-channel NMOSFETs subjected to a series of high-field stresses for the highfrequency, low-frequency, and quasi-static frequency measurements, respectively. It is indicated that short-channel devices show more serious distortions and may suffer a more nonuniform distribution of stress current than long-channel devices. This phenomenon may be attributed to the crowding of gate tunneling currents induced by the geometric effect, and it finally 


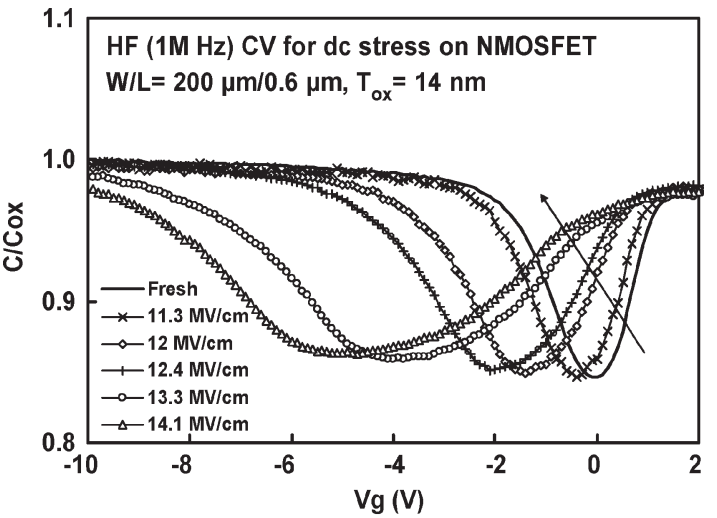

(a)

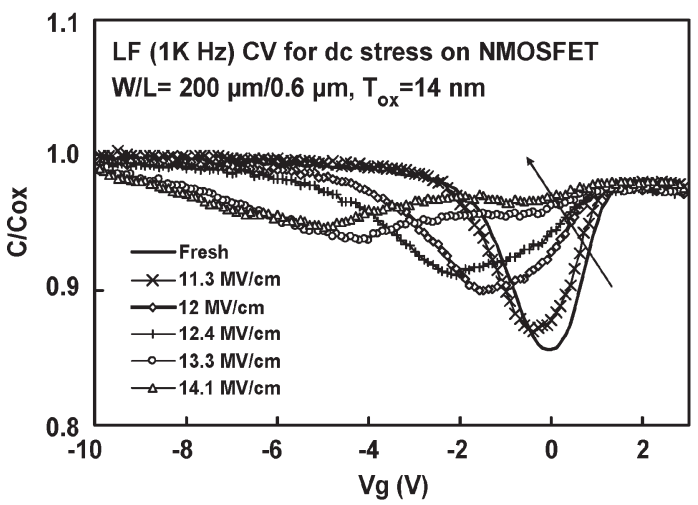

(b)

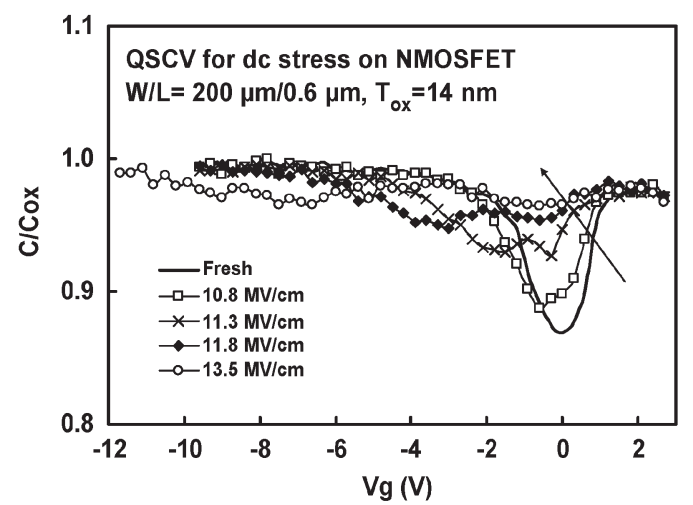

(c)

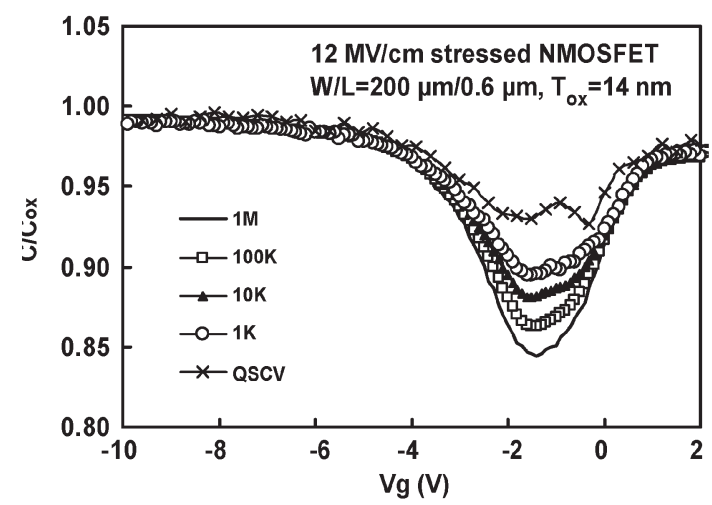

(d)

Fig. 3. (a) High-frequency (1 MHz), (b) low-frequency (1 kHz), and (c) quasistatic $C-V$ characteristics of short-channel $(0.6 \mu \mathrm{m})$ NMOSFETs subjected to a series of dc high-field stresses. (d) Comparison of $C-V$ curves over the high, low, and quasi-static frequencies.

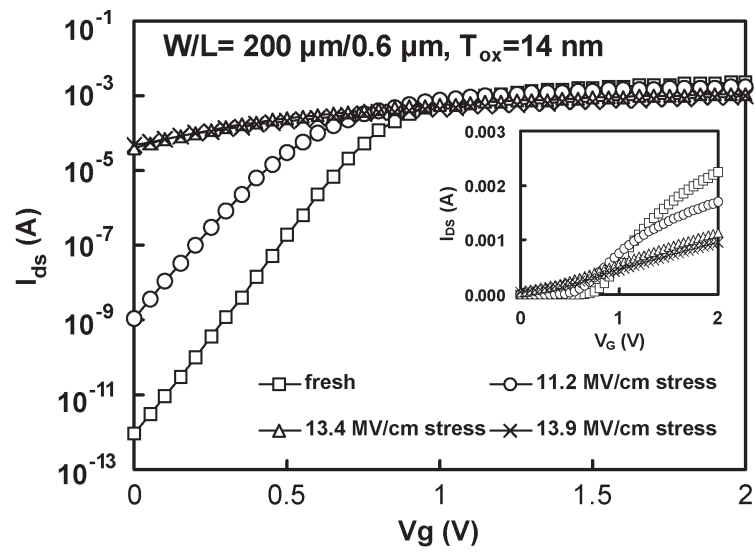

(a)

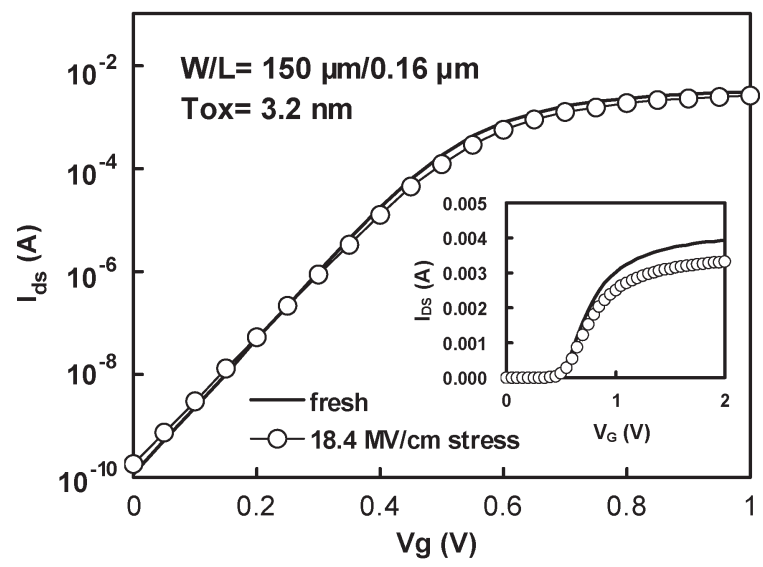

(b)

Fig. 4. Subthreshold $I-V$ characteristics of (a) thick-oxide and (b) thin-oxide transistors subjected to heavy stresses prior to oxide breakdown. The inserts are the plots in linear scale, which can clearly indicate the increase of leakage currents and the decrease of conduction currents.

causes the nonuniform distribution of trapped charges. Fig. 3(d) shows the comparison of $C-V$ curves over the high, low, and quasi-static frequencies. It is observed that the lower the frequency is, the larger the distortion and LNU effect become.

To investigate the impact on characteristics of MOSFETs subjected to gate oxide stresses, the current-voltage characteristics and charge-pumping current were measured. Fig. 4(a) and (b) shows the subthreshold $I-V$ characteristics of thickand thin-oxide transistors subjected to the heavy stresses prior to oxide breakdown. The inserts are the plots in linear scale, which clearly indicate the increase of leakage currents and the decrease of conduction currents. As we can see, the thick-oxide device shows a lot of degradation both in the subthreshold swing and conducting current. On the contrary, the thin-oxide device shows a less degradation than the thick-oxide device. This may be due to the fact that the thick-oxide transistor has a larger number of stress-induced traps than the thin-oxide transistors.

The charge-pumping technique is better suited to study the nonuniform spatial distribution of interface and border traps in a MOSFET, as compared with the subthreshold $I-V$ measurement. Fig. 5(a) and (b) shows the charge-pumping characteristics of NMOSFETs subjected to high-field stresses, where the gate oxide thickness is $14 \mathrm{~nm}$ and the channel lengths are 10 and 


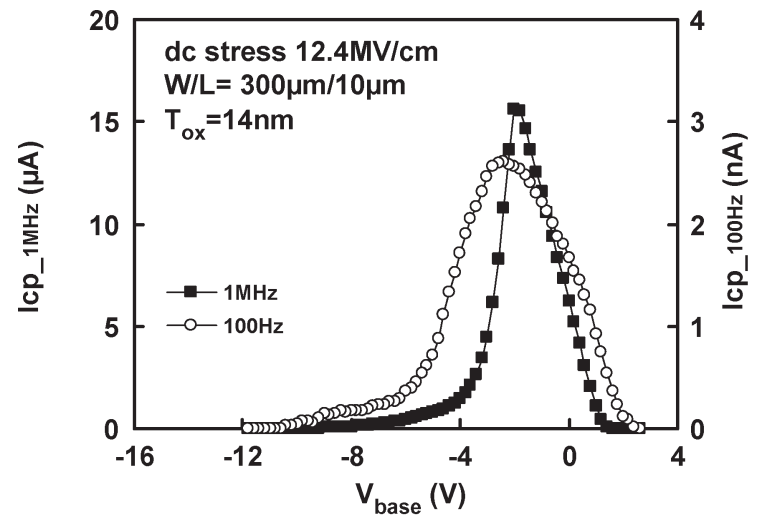

(a)

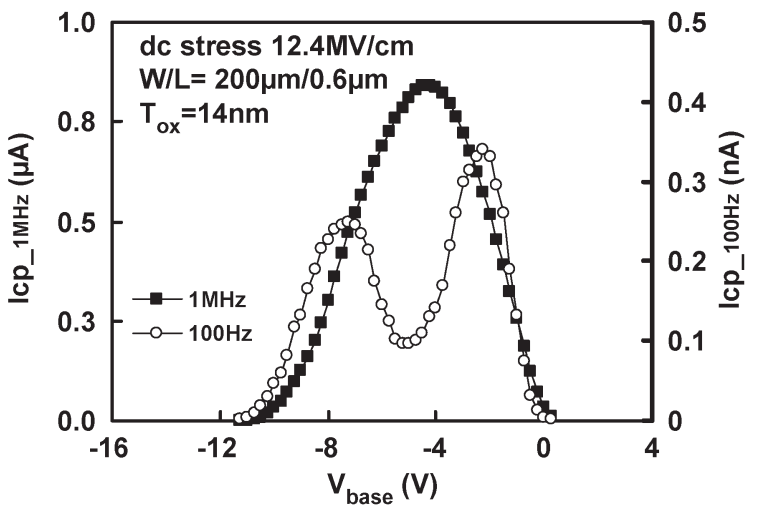

(b)

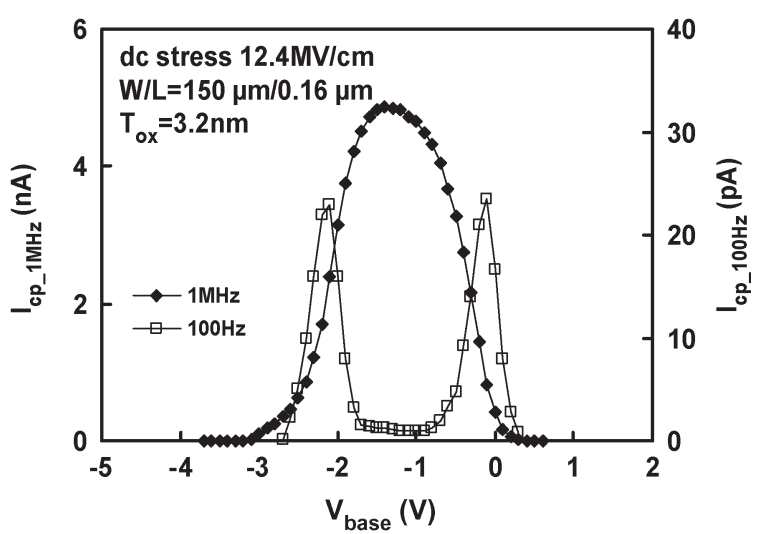

(c)

Fig. 5. One-megahertz and $100-\mathrm{Hz}$ charge-pumping characteristics of (a) long-channel $(10 \mu \mathrm{m})$ and (b) short-channel $(0.6 \mu \mathrm{m})$ NMOSFETs with a 14-nm gate oxide subjected to dc high-field stresses. (c) Charge-pumping characteristics of a short-channel $(0.16 \mu \mathrm{m}) 3.2-\mathrm{nm}$ gate oxide NMOSFET subjected to high-field dc stresses.

$0.6 \mu \mathrm{m}$, respectively. Fig. 5(c) shows the charge-pumping characteristics of an NMOSFET subjected to the high-field stresses, where the gate oxide thickness is $3.2 \mathrm{~nm}$ and the channel length is $0.16 \mu \mathrm{m}$. As the figures show, the doublepeak $I_{\mathrm{cp}}$ only occurs for the short-channel MOSFETs under the low-frequency measurements. Furthermore, under the same stress field, the double-peak phenomenon of $I_{\mathrm{cp}}$ is more obvious for the larger width-to-length ratio and thinner oxide device. Fig. 6(a) and (b) shows the high- and low-frequency charge-pumping characteristics of a short-channel NMOSFET subjected to a series of dc stresses before oxide breakdown. As

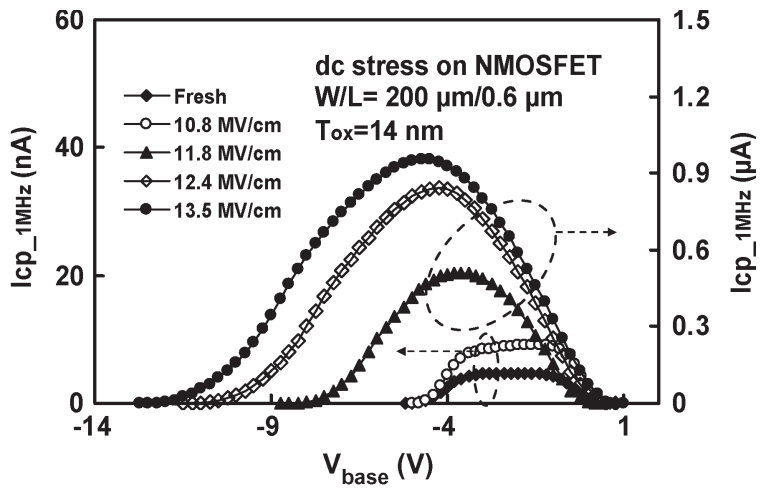

(a)

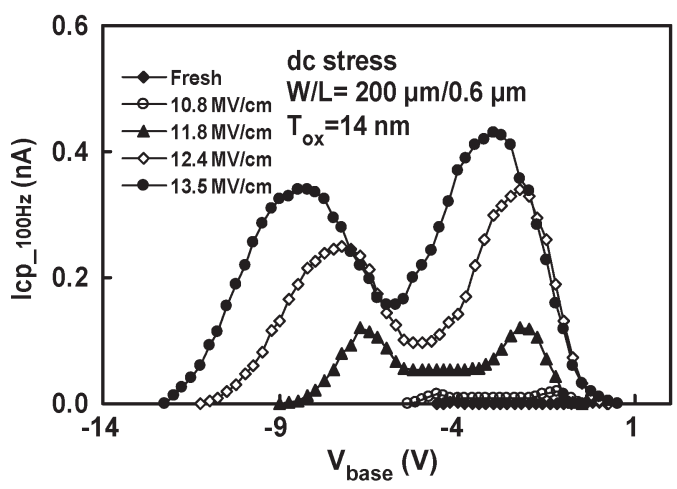

(b)

Fig. 6. (a) High-frequency $(1 \mathrm{MHz})$ and (b) low-frequency $(100 \mathrm{~Hz})$ chargepumping characteristics of a short-channel $(0.6 \mu \mathrm{m})$ NMOSFET subjected to a series of dc stresses before oxide breakdown.

can be seen in high-frequency measurements, $I_{\mathrm{cp}}$ is increasing, which is accompanied by a curve shift to the lower $V_{\text {base }}$. This implies that a lot of donor-like interface traps were induced [17]. On the other hand, in the low-frequency measurements, the left peak $V_{\mathrm{base}}$ of $I_{\mathrm{cp}}$ decreases with the increasing stress field. The nonuniform effect of the low-frequency measurement is more serious than that of the high-frequency measurement. For the geometric effect, the cause of the nonuniform phenomenon may be due to the current crowding and filament under a high-field stress, which leads to a localized injection of avalanche hot carriers and causes impact ionization within the oxide. Therefore, a higher stress field (or thinner oxide) leads to a more pronounced nonuniformity issue. Additionally, for the oxide thickness effect, the cause may be due to the different tunneling mechanisms of the thick $(14 \mathrm{~nm})$ and thin oxides $(3.2 \mathrm{~nm})$. Generally, the current transport mechanisms are trapassisted tunneling and direct tunneling for the thick and thin oxides, respectively. The direct tunneling may be prone to a current filament and has a worse nonuniformity issue than the trap-assisted tunneling.

During the charge-pumping measurement, $I_{\mathrm{cp}}$ increases when the top-level voltage $V_{\text {top }}$ of the applied signal is larger than the flatband voltage $V_{\mathrm{FB}}$ of the NMOSFET, and $I_{\mathrm{cp}}$ decreases when the base-level voltage $V_{\text {base }}$ of the applied signal is larger than the flatband voltage $V_{\mathrm{FB}}$ of the NMOSFET. Hence, the lower $V_{\mathrm{FB}}$ is, the lower the voltage of the applied signal to generate $I_{\mathrm{cp}}$ will be. As reported [17], FowlerNordheim injection of electrons from the gate $\left(V_{g}<0\right)$ 


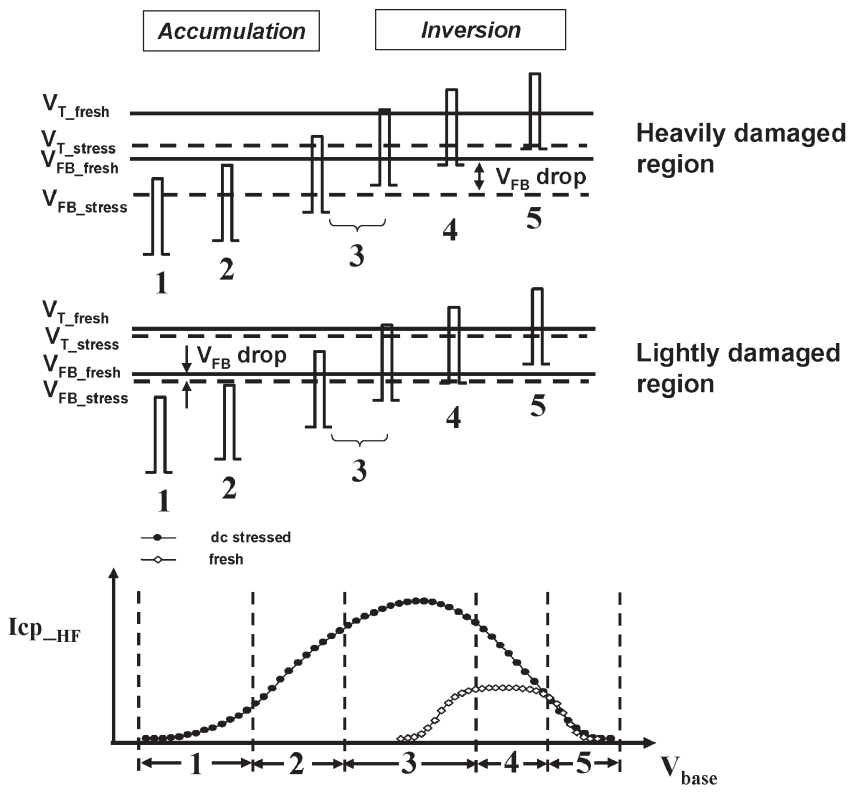

(a)
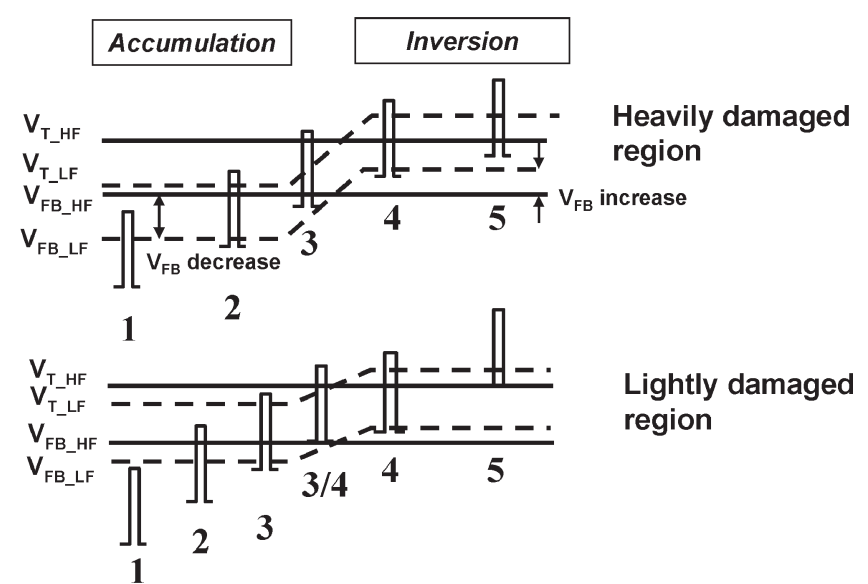

Lightly damaged region

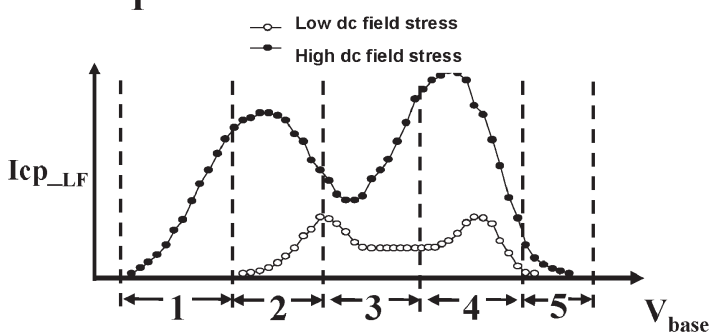

(b)

Fig. 7. Schematics of the LNU mechanism under (a) high-frequency and (b) low-frequency charge-pumping measurements for MOSFETs after highfield dc stresses.

generates a lot of donor-like interface traps and positive charges in the bulk of the oxide or at the $\mathrm{Si}-\mathrm{SiO}_{2}$ interface. $V_{\mathrm{FB}}$ and $V_{T}$ are therefore lowered by the contribution of $Q_{\text {eff }}$. Fig. 7(a) suggests the schematic diagram of the LNU mechanism under high-frequency measurements for a high-field-stressed NMOSFET. After a high-field stress, both lightly and heavily damaged regions exist in the whole gate area due to the nonuniform distribution of stress currents. The heavier damaged oxide provides a larger number of traps and leads to a larger $Q_{\text {eff. }}$. Therefore, different amounts of $Q_{\text {eff }}$ are induced, corresponding to the different extents of damages. From the experimental data, it is suggested that a larger $Q_{\text {eff }}$ in a more heavily damaged region can further lower the $V_{\mathrm{FB}}$ and $V_{T}$ values of the high-frequency $C-V$ curves, which can lead to the split of $V_{\mathrm{FB}}$ and $V_{T}$ inside the whole MOSFET. The MOSFET can be thought of as being composed of several parts in parallel with different $V_{\mathrm{FB}}$ 's, $V_{T}$ 's, and concentrations of interface traps, and the resultant charge-pumping signal is the sum of the charge-pumping signals of the composing parts. When the high-frequency charge-pumping signal is applied to the MOSFET with the constant amplitude and increasing base voltage, a wide distribution of high $I_{\mathrm{cp}}$ can be induced across the transition region between different damaged regions. Fig. 7(b) proposes the schematic diagram of the enhanced LNU mechanism under low-frequency measurements for an NMOSFET after high-field stresses. Under low-frequency measurements below $1000 \mathrm{~Hz}$, the donor-like border traps can contribute extra positive trapped charges and further lower the $V_{\mathrm{FB}}$ and $V_{T}$ values in the accumulation regime, as compared with the high-frequency measurement. Furthermore, the acceptorlike border traps contribute extra negative trapped charges and further elevate $V_{\mathrm{FB}}$ and $V_{T}$ in the inversion regime. Hence, during the low-frequency measurements, the changing biased voltage causes the abrupt splits of $V_{\mathrm{FB}}$ and $V_{T}$ between the accumulation and inversion regimes. This pronounced LNU effect finally leads to the double-peak $I_{\mathrm{cp}}$. Additionally, by the superposition of $I_{\mathrm{cp}}$ of heavily and lightly damaged regions, the different amplitudes of current peaks can be explained for the high field stress curve.

\section{CONCLUSION}

The deterioration induced by the nonuniform-distributed traps is studied for MOSFETs subjected to high-field stresses. According to the experimental data, the low-frequency drift of device characteristics is enhanced and more serious than that of the high frequency. The cause may be due to the extra contribution of border-trapped charges under low-frequency operation. Furthermore, a more severe nonuniform damage is found in the shorter-channel device, which is possibly due to the crowding of tunneling currents. Additionally, a thinner oxide or higher stress field may help to form a current filament to deteriorate the nonuniformity by the localized injection of avalanche hot carriers and impact ionization. Therefore, precautions against drifts of characteristics should be taken for the design of sensitive analog circuits under the low-frequency operation.

\section{REFERENCES}

[1] R. Thewes, R. Brederlow, C. Schlunder, P. Wieczorek, A. Hesener, B. Ankele, P. Klein, S. Kessel, and W. Weber, "Device reliability in analog CMOS applications," in IEDM Tech. Dig., 1999, pp. 81-84.

[2] P. R. Kinget, "Device mismatch and tradeoffs in the design of analog circuits," IEEE J. Solid-State Circuits, vol. 40, no. 6, pp. 1212-1224, Jun. 2005.

[3] M. S. Liang, C. Chang, Y. T. Yeow, C. Hu, and R. W. Brodersen, "MOSFET degradation due to stressing of thin oxide," IEEE Trans. Electron Devices, vol. ED-31, no. 9, pp. 1238-1244, Sep. 1984.

[4] R. S. Scott and D. J. Dumin, "The charging and discharging of highvoltage stress-generated traps in thin silicon oxide," IEEE Trans. Electron Devices, vol. 43, no. 1, pp. 130-136, Jan. 1996. 
[5] J. G. Hwu and W. S. Wang, "Direct indication of lateral non-uniformities of MOS capacitors from the negative equivalent interface trap density based on charge-temperature technique," Appl. Phys. A, Solids Surf., vol. 40, no. 1, pp. 41-46, May 1986.

[6] S. W. Huang and J. G. Hwu, "Lateral non-uniformity of effective oxide charges in MOS capacitors with $\mathrm{Al}_{2} \mathrm{O}_{3}$ gate dielectrics," IEEE Trans. Electron Devices, vol. 53, no. 7, pp. 1608-1611, Jul. 2006.

[7] W.-K. Chim and P.-S. Lim, "Latent damage investigation on lateral nonuniform charge generation and stress-induced leakage current in silicon dioxide subjected to high-field current impulse stressing," IEEE Trans. Electron Devices, vol. 47, no. 2, pp. 473-481, Feb. 2000.

[8] W. K. Chim and P. S. Lim, "Hole injection with limited charge relaxation, lateral non-uniform hole trapping, and transient stress-induced leakage current in impulse-stressed thin $(<5 \mathrm{~nm})$ nitrided oxides," J. Appl. Phys., vol. 91, no. 3, pp. 1304-1313, 2002.

[9] D. M. Fleetwood, "Border traps in MOS devices," IEEE Trans. Nucl. Sci., vol. 39, no. 2, pp. 269-271, Apr. 1992.

[10] D. M. Fleetwood, "Fast and slow border traps in MOS devices," IEEE Trans. Nucl. Sci., vol. 43, no. 3, pt. 1, pp. 779-786, Jun. 1996.

[11] R. E. Paulsen and M. H. White, "Theory and application of charge pumping for the characterization of $\mathrm{Si}-\mathrm{SiO}_{2}$ interface and near-interface oxide traps," IEEE Trans. Electron Devices, vol. 41, no. 7, pp. 1213-1216, Jul. 1994.

[12] N. L. Cohen, R. E. Paulsen, and M. H. White, "Observation and characterization of near-interface oxide traps with $C-V$ techniques," IEEE Trans. Electron Devices, vol. 42, no. 11, pp. 2004-2009, Nov. 1995.

[13] C. E. Weintraub, E. Vogel, J. R. Hauser, N. Yang, V. Misra, J. J. Wortman, J. Ganem, and P. Masson, "Study of low-frequency charge pumping on thin stacked dielectrics," IEEE Trans. Electron Devices, vol. 48, no. 12, pp. 2754-2762, Dec. 2001.

[14] C. Y. Lu, K. S. Chang-Liao, P. H. Tsai, and T. K. Wang, "Depth profiling of border traps in MOSFET with high- $\kappa$ gate dielectric by charge-pumping technique," IEEE Electron Device Lett., vol. 27, no. 10, pp. 859-862, Oct. 2006.

[15] J. S. Brugler and P. G. A. Jespers, "Charge pumping in MOS devices," IEEE Trans. Electron Devices, vol. ED-16, no. 3, pp. 297-302, Mar. 1969.

[16] G. Groesenken, H. E. Maes, N. Beltran, and R. F. De Keersmaecker, "A reliable approach to charge-pumping measurements in MOS transistors," IEEE Trans. Electron Devices, vol. ED-31, no. 1, pp. 42-53, Jan. 1984.

[17] P. Heremans, J. Witters, G. Groesenken, and H. E. Maes, "Analysis of the charge pumping technique and its application for the evaluation of MOSFET degradation," IEEE Trans. Electron Devices, vol. 36, no. 1, pp. 1318-1335, Jul. 1989.

[18] C. Leroux, P. Andreucci, and G. Reimbold, "Analysis of oxide breakdown mechanism occurring during ESD pulses," in Proc. IEEE IRPS, 2000, pp. 276-282.

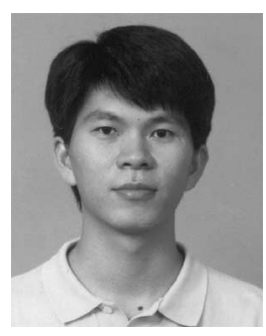

Jen-Chou Tseng received the B.S. degree from National Chiao-Tung University, Hsinchu, Taiwan, R.O.C., in 1992, the M.S. degree from National Tsing-Hua University, Hsinchu, in 2000, and the $\mathrm{Ph} . \mathrm{D}$. degree from National Taiwan University, Taipei, Taiwan, in 2007.

$\mathrm{He}$ is currently with Taiwan Semiconductor Manufacturing Company, Hsinchu, as a Manager in the ESD/EOS engineering.

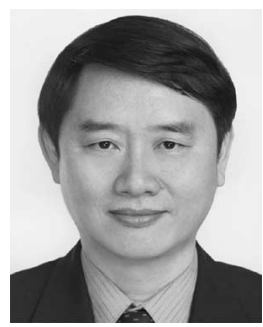

Jenn-Gwo Hwu (M'93-SM'99) was born in Tainan, Taiwan, R.O.C., on August 29, 1955. He received the B.S. degree in electronic engineering from National Chiao-Tung University, Hsinchu, Taiwan, in 1977 and the M.S. and Ph.D. degrees in electrical engineering from National Taiwan University, Taipei, Taiwan, in 1979 and 1985, respectively.

He joined the faculty of National Taiwan University in 1981. He is currently a Professor with the Department of Electrical Engineering and the Graduate Institute of Electronics Engineering, National Taiwan University. From 1997 to 1998, he was the Vice Chairman of the Department of Electrical Engineering, National Taiwan University. From February 2004 to January 2006, he was invited as the Dean of the College of Electrical Engineering and Computer Science, National United University, Miaoli, Taiwan. In December 2005, he was invited as the Coordinator of the Micro-Electronics Engineering Program, Department of Engineering and Applied Sciences, National Science Council, Taiwan. In August 2006, he was appointed as a Distinguished Professor of the National Taiwan University. In August 2007, he was appointed as the Chairperson of the Department of Electrical Engineering, National Taiwan University. He has taught courses on circuits, electronics, solid-state electronics, semiconductor engineering, MOS capacitor devices, radiation effects on MOS system, and oxide reliability. His research interests include ultrathin gate oxide and Si MOS devices.

Dr. Hwu qualified as a Licensed Professional Technique Expert on Electrical and Electronics Engineering, Taiwan, in 1978 and 1980, respectively. He received the Outstanding Teaching Award from the Ministry of Education in 1991 and from National Taiwan University in 1987 and 2003, the Excellent Teaching Award from the College of Engineering, National Taiwan University, in 1988, 1989, 1990, 1991, and 1993 and from National Taiwan University in 1999, 2000, and 2002, the Jan Ten-You Paper Award from the Chinese Institute of Engineering, Taiwan, in 1999, and the Scientific Paper Award from the Far Eastern Y. Z. Hsu Science and Technology Memory Foundation, Taiwan, in 2005. 